\title{
Rapid Behavior Adaptation for Human-centered Robots based on Integration of Primitive Confidence on Multi-sensor Elements
}

\author{
Saifuddin Md. Tareeq and Tetsunari Inamura \\ The Graduate University for Advanced Studies, National Institute of Informatics, Japan \\ smtareeq@nii.ac.jp;inamura@nii.ac.jp
}

\begin{abstract}
This paper presents a rapid learning method of behavior policy for mobile robots teleoperated by an operator. Rapid policy adaptation cannot be achieved when data from every process cycle is used for learning because significant data, which have a major effect on learning, are not differentiated with insignificant data. We propose a method to solve the problem by selecting significant data for the learning based on change in degree of confidence for each sensor element. A small change in the degree of confidence can be regarded as reflecting insignificant data for learning, so that data can be discarded. Accordingly the system can avoid having to store too much experience data and the robot can adapt rapidly to changes in the user's policy. In this paper we discuss the experimental result of an experiment in which significance evaluation is carried out on each proposition of each sensor. And in the experiment user policy changes between 'avoid' and 'approach' on a mobile robot.
\end{abstract}

Keywords: Bayesian Network, Rapid Adaptation, Degree of Confidence

\section{Introduction}

One of the important abilities for personal service robots which act in real environment with human beings is to learn and acquire novel behavior strategy according to observation of users behavior. To learn the behavior strategy, conventional methods observes sets of sensor input and command output, extracts meaningful relation between the sensor and commands using statistical methods. But the performance of the learning strongly depends on the quality of the dataset of sensor and command. When the dataset included meaningful data, the learning would be a success; however it is difficult to obtain meaningful experience data for human-robot interaction in real world, because the robots basically stores the data in every process cycle. For example, when a user kept operating same command in same situation, the statistical learning procedure tends to output the frequent command even though the sensor is not the frequent but rare. To select the rare command for rare sensor, the system should ignore insignificant frequent dataset to avoid bad learning quality. In this paper, we propose a technique to manage experience dataset with evaluation of significance of the dataset based on a concept of change in degree of confidence for each proposition of each sensor. A small change can be regarded as an insignificant data for learning, so that data will be discarded. Accordingly, the system can avoid having to store too frequent experience data.
Conventional methods like window [1][2] based adaptation require background investigation of the domain to find a suitable window, dual model [3] [4] based methods uses separate model for short term and long term learning but are unsuitable for rapid adaptation with long term model, interaction [5][6][7][8] based methods does not deal with adaptation with user policy but only with acquiring user policy.

\section{The problem}

From the above discussion it is evident that by using huge number of data for learning it is difficult to adapt rapidly to follow user preference. And for robotic application rapid adaptation to user policy is desirable because otherwise user may be bored easily. Therefore we have chosen Bayesian network to represent preference, because it can represent degree of confidence for behavior decision as probability and can interact with user with its internal state and can handle uncertainty associated with observation.

A Bayesian network is a directed acyclic graph consists of parent nodes representing causes and child nodes representing effects as shown in Fig.1. Each node can represent a multi valued variable, comprising of collection of a mutually exclusive propositions. Let the variable be labeled by capital letters $\left(\mathrm{X}, \mathrm{Y}, Z_{1}\right.$, $\left.Z_{2}, Z_{3}\right)$ and their possible values by the corresponding lowercase letters $\left(\mathrm{x}, \mathrm{y}, z_{1}, z_{2}, z_{3}\right)$. Each directed link 


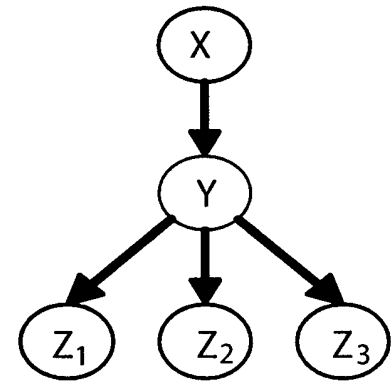

Figure 1: A Bayesian network.

$X \rightarrow Y$ is quantified by a fixed conditional probability table $C P T$ in which the $(\mathrm{x}, \mathrm{y})$ entry is given by

$C P T_{(Y \mid X)} \equiv\left(\begin{array}{cccc}P\left(y_{1} \mid x_{1}\right) & P\left(y_{2} \mid x_{1}\right) & \ldots & P\left(y_{m} \mid x_{1}\right) \\ P\left(y_{1} \mid x_{2}\right) & P\left(y_{2} \mid x_{2}\right) & \ldots & P\left(y_{m} \mid x_{2}\right) \\ \vdots & \vdots & \ddots & \vdots \\ P\left(y_{1} \mid x_{l}\right) & P\left(y_{2} \mid x_{l}\right) & \ldots & P\left(y_{m} \mid x_{l}\right)\end{array}\right)$

The $C P T$ is calculated for all parent-child nodes. The reasoning can be expressed as

$$
\operatorname{Bel}(Y)=\beta \lambda(Y) \pi(Y),
$$

where $\lambda(Y)$ represents the current strength of diagnostic support contributed by the children of $Y$ given by $\prod_{i} \lambda_{i}(Y), \pi(Y)$ represent the current strength of the causal support contributed by the parents of $Y$ and $\beta$ is the coefficient for normalization [9]. Elements of $\operatorname{Bel}(Y)$ indicate the plausibility for each proposition of a node. When $Y$ does not have any parent, $\pi(Y)$ is the prior probability of $Y$. The likelihood vector $\lambda_{i}(Y)$ is calculated as

$$
\lambda_{i}(Y)=C P T_{\left(Z_{i} \mid Y\right)} \lambda\left(z_{i}\right),
$$

where $\operatorname{CPT}_{\left(Z_{i} \mid Y\right)}$ quantifies $Y \rightarrow Z_{i}$ link and $\lambda\left(z_{i}\right)$ is the observed input of $Z_{i}$. One of the advantages of Bayesian networks is that a robot can evaluate the vagueness of a behavior decision, and this leads it to ask questions and give suggestions to users [5]. For example, the robot should ask the user to confirm the behavior decision when the elements of $\operatorname{Bel}(\mathrm{Y})$ are almost equal.

We emphasis the problem by explaining the reason about the need of the change in degree of confidence based significance evaluation of data with an example as shown by Fig.2. The robot observes a user preference and gathers sensor information continuously. The user's preference is given by the behavior decision. The robot observes the user's behavior $b_{j}$ and gathers the sensor information $d_{i}$ at the same moment and represented as data $D[t]=\left\{b_{j}, d_{i}\right\}$. Let $N$ be the number of observed data, $N_{j}$ be number of observations of behavior $b_{j}$, and $n_{i j}$ be the number of obser-

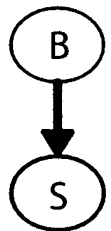

Figure 2: A simple Bayesian network.

vation of behavior $b_{j}$ when $d_{i}$ is observed. One of the simplest calculations based on the observation is

$$
\begin{gathered}
P\left(d_{i} \mid b_{j}\right)=P\left(S=d_{i} \mid B=b_{j}\right)=\frac{n_{i j}}{N_{j}}, \\
P\left(b_{j}\right)=P\left(B=b_{j}\right)=\frac{N_{j}}{N},
\end{gathered}
$$

A problem arises with this simple calculation when the data is continuously input during the observation. Suppose that the user preference changes between two behaviors, $b_{1}$ and $b_{2}$. When a rare but important behavior $b_{2}$ is observed while $N_{2} \ll N$, the probability $P\left(B=b_{2}\right)$ is close to 0 and $P\left(B=b_{1}\right)$ is close to 1. This simple prior probability calculation by $\mathrm{Eq}(5)$ based on frequency of the number of data causes the problem because even if the conditional probability by $\mathrm{Eq}(4)$ shows a feasible value, the degree of confidence, $\operatorname{Bel}(B)$, by $\mathrm{Eq}(2)$ becomes heavily biased because of the prior probability calculation by $\mathrm{Eq} 5$. Consequently the system tends to output the most frequent command even though sensor input for rare behavior is given. This factor also causes another problem that the robot cannot adapt rapidly to changeable preference of the user.

\section{The method}

The change in degree of confidence based significance evaluation is described in this section. We use a Dirichlet distribution to evaluate the significance of data based on changes in the degree of confidence. A m-directional Dirichlet distribution for $\mathbf{w}=\left\{w_{1}, w_{2}, \ldots, w_{m}\right\}$, is given by

$$
f_{d}\left(\mathbf{w} ; \alpha_{1}, \ldots, \alpha_{m}\right)=\frac{1}{Z} \prod_{k} w_{k}^{\alpha_{k-1}},
$$

where,

$$
Z=\frac{\prod_{k-1}^{m} \Gamma\left(\alpha_{k}\right)}{\Gamma\left(\sum_{k-1}^{m} \alpha_{k}\right)},
$$

is a normalization factor, $\Gamma$ is the gamma function and the $m$ parameters $\alpha_{m}$ are assumed to be positive. The distribution parameter $\alpha_{i}$ is expressed as $\alpha_{i}=1+n_{i j}$. The system increases $\alpha_{i}$ according to the observed sensor proposition. When $\alpha_{i}$ becomes larger than the other Dirichlet parameters, the peak of the distribution moves within a small area at the 


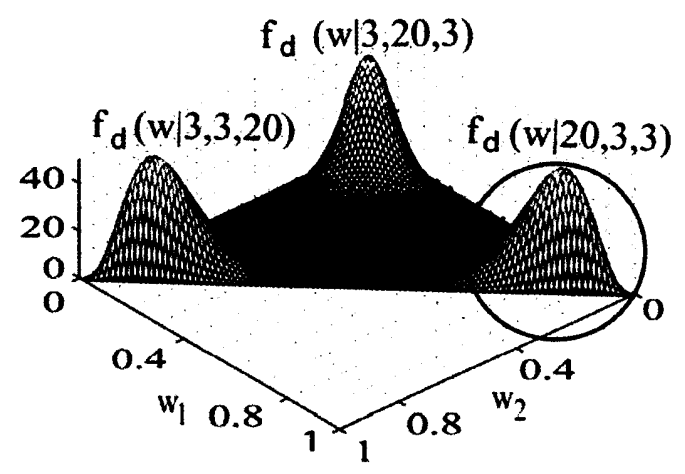

Figure 3: Dirichlet density functions with peak moved to the corresponding parameter.

end of corresponding variable as shown by Fig.3. The system calculates the degree of confidence at $t-1$ and $t$. Confidence at time $t$ is calculated as

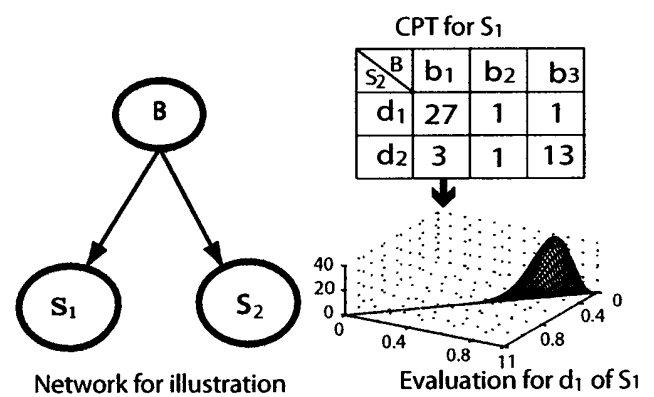

Figure 4: Evaluation of significance and CPT for each sensor. Here $d_{i} \epsilon\left\{d_{1}, d_{2}\right\}$ and $b_{i} \epsilon\left\{b_{1}, b_{2}, b_{3}\right\}$.

$$
C_{i}[t]=\int_{\Delta_{i}} f_{d}(\mathbf{w} ; \alpha[t]) d \mathbf{w}
$$

where $f_{d}(\mathbf{w} ; \alpha[t])$ is the Dirichlet distribution at time $t$ and $\Delta_{i}$ represents area of integration where the peak of the distribution is moved by observing sensor proposition $d_{i}$ as shown by the circle in Fig. 3. The change in the two consecutive degrees of confidence can be regarded as the importance of the observation to the learning process. To evaluate the significance of the observation data, the criteria

$$
E_{i}=\left|C_{i}[t]-C_{i}[t-1]\right|,
$$

is calculated. Here $C_{i}$ and $E_{i}$ are calculated when the sensor proposition $d_{i}$ is observed. When $E_{i} \geq \theta$, data $D[t]$ are significant for learning and accepted, that is, $n_{i j}^{s i g}:=n_{i j}^{\text {sig }}+1$, and data are insignificant for learning and discarded when $E_{i}<\theta$. This sensor proposition level significance evaluation process is shown in Fig. 4. Let $n_{i j}^{\text {sig }}$ be the number of significant observations while the sensor proposition $d_{i}$ is observed with behavior $b_{j}$ and $N_{j}^{s i g}$ be the number of significant observation of behavior $b_{j}$. Then we get

$$
\begin{gathered}
P\left(d_{i} \mid b_{j}\right)=P\left(S=d_{i} \mid B=b_{j}\right)=\frac{n_{i j}^{s i g}}{N_{j}^{s i g}}, \\
P\left(b_{j}\right)=\frac{N_{j}^{s i g}}{\sum_{j} N_{j}^{s i g}}
\end{gathered}
$$

Now because of the significance evaluation of senor information when user policy changes between two behaviors, $b_{1}$ and $b_{2}$, even if a rare but important behavior $b_{2}$ is observed much smaller number than behavior $b_{1}, N_{2}^{s i g}$ can not be much smaller than $N_{1}^{s i g}$. Therefore, the degree of confidence remain unbiased and it becomes easier to adjust rapidly to the user's new policy with a few observations.

\section{Experiment and Result}

\subsection{Experimental Setup}

We developed a teaching and learning system in a virtual environment that incorporated our concept. The environment was prepared using webot real-time simulation software as shown in Fig. 5. The environment had an enclosed area of $8[\mathrm{~m}] \times 8[\mathrm{~m}]$. A static square obstacle whose size was $1[\mathrm{~m}] \times 1[\mathrm{~m}]$ was placed inside the area. The user interface consisted of a lever joystick and the user controlled the robot by using it. We taught two policies to the robot, avoid and approach, in the field. In the experiment, we used a Bayesian network consists of eight distance sensor nodes and a behavior node as in Fig. 5. The robot model had

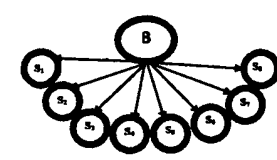

Exp. network

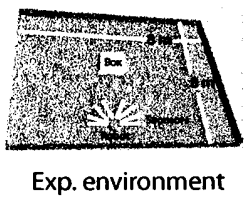

Exp. environment
Figure 5: A Bayesian network and environment used in the experiment.

eight front laser distance sensors $\left(S_{i}, i=1,2, \ldots, 8\right)$ mounted on the front to measure the distance to obstacles along a horizontal line parallel to the floor. Joystick inputs were translated into discrete instructions by using a predetermined threshold. We found [10] that area of integration was inversely proportional and threshold value was directly proportional to the time required reach the evaluation criteria respectively. Therefore we set the area of integration to the maximum non-overlapping area and the threshold to $1.0 \times 10^{-6}$. 
The user can teleoperate the robot at any time and halt operation temporarily for changing robot orientation in the virtual environment. When user do not operate the robot, it operates automatically with it's own degree of confidence for behavior node. Previously we have shown that our algorithm can adapt to the user preference rapidly [10]. In that paper we have shown that robot adapted to user preference like go forward $\left(b_{1}\right)$, turns left $\left(b_{2}\right)$ or turn $\operatorname{right}\left(b_{3}\right)$. In this experimental setup user policy correspond to robot behavior, avoids and approach. Avoid policy is accomplished by going forward when there is no obstacle and turning left when there is an obstacle. Approach policy is accomplished by going forward when there is no obstacle and approaching the obstacle when there is one.

\subsection{Experimental Results}

The user first taught avoid policy. Then the user changed the policy to approach. Fig. 6 shows a snapshot of the CPT for sensor number 5 and number of data evaluated as significant and kept in secondary database for that particular sensor. From the numbers in the CPT we can easily recognize that only the significant data is stored in the secondary database for each proposition of each sensor. Flat part of the graph represents when data is evaluated as insignificant and discarded or the robot operate automatically. From the CPT we can also recognize that policy overriding is done by accepting data for different sensor propositions. Policy adaptation is accomplished through the integration of all significant data for each proposition of each sensor. Fig. 7 shows the changes in prob-

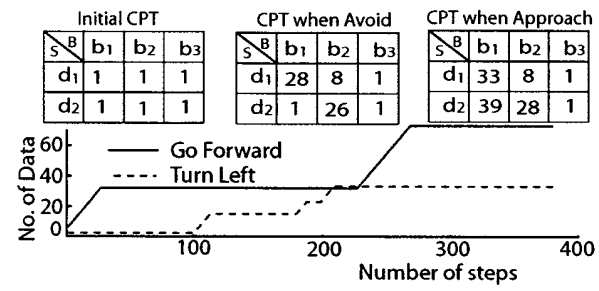

Figure 6: CPT and Number of data in the secondary database during policy adaptation for sensor node 5 .

ability of degree of confidence during teleoperation. This is integrated probability over all sensor elements. Robot could rapidly adapted to the new policy around 320 by overriding the previous policy.

\section{Conclusions}

Experimental results show that proposed method can adapt to user's policy change based on significance evaluation using change in degree of confidence. The novel point of the method is that the policy adaption

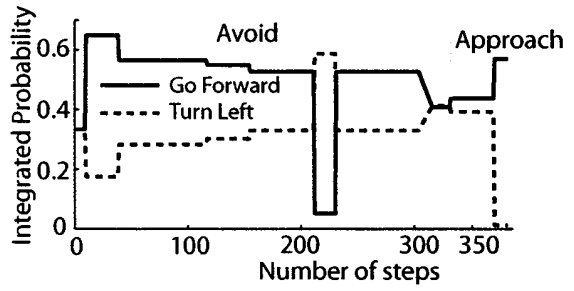

Figure 7: Integrated probability of behavior during policy adaptation.

depends on the number of selected significant data rather than enormous amount of observed data. In this paper we have described the result of significance evaluation for each sensor proposition of every sensor node. The result confirms that that only significant sensor observation is enough for rapid adaptive learning. In the near future we would like to investigate the performance of our algorithm in discovering the change in the context of experimental environment.

\section{References}

[1] Widmer G. and Kubat M., Learning in the Presence of Concept Drift and Hidden Contexts in Machine Learning, 23, 69101, 1996.

[2] Klinkenberg R., Learning Drifting Concepts: Example selection vs. Example weighting in Intelligent Data Analysis, Special Issue on Incremental Learning Systems Capable of Dealing with Concept Drift, 8(3), 281-300, 2004.

[3] Billsus D. and Pazzani M. J., User Modeling for Adaptive News Access in User Modeling and User-Adapted Interaction, News Access in U
$10,147-180,2000$.

[4] Chiu P. and Webb G., Using Decision tree for Agent Modeling; Improving Prediction Performance in User Modeling and User-Adapted Interaction, 8, 131-152, 1998.

[5] Inamura T., Inaba M. and Inoue H., PEXIS: Probabilistic Experience Representation Based Adaptive Interaction Sys tem for Personal Robots in Systems and Computers in Japan, 35(6), 98-109, 2004

[6] Inamura T., Inaba M. and Inoue H., User Adaptation of Human-Robot Interaction Model based on Bayesian Network and Introspection of Interaction Experience in Proc. of Int'1 Conf. on Intelligent Robots and Systems (IROS 2000), 2139$2144,2000$.

[7] Inamura T., Inaba M. and Inoue H., Acquisition of Probabilistic Decision Model based on interactive teaching method in proceedings of the ninth International Conference on Advanced Robotics, ICAR, 523-528, 1999.

[8] Nicolescu M. and Mataric M., Learning and interacting in human-robot domains in IEEE Transactions on System in Man and Cybernetics-Part A: Systems and Humans, 31(5), 419-430, 2001

[9] Pearl J., Probabilistic reasoning in Intelligent system; network of plausible inference, Second Edition, Morgan Kaufman, 1988.

[10] Tareeq S. M. and Inamura T., A sample discarding strategy for rapid adaptation to new situation for Bayesian behavior learning in Proceedings of the IEEE International Conference on Robotics and Biomemtics, 1950-1955, 2008. 